\title{
Lesión compleja del codo pediátrico: fractura de cóndilo externo asociada a luxación posteromedial de codo.
}

\author{
DOI: http//dx.doi.org/10.37315/SOTOCAV20202825556
}

DARÁS-BALLESTER A, JOVER-JORGE N, DOMÉNECH-FERNÁNDEZ P.

SERVICIO DE CIRUGÍA ORTOPÉDICA Y TRAUMATOLOGÍA. HOSPITAL UNIVERSITARIO Y POLITÉCNICO LA FE. VALENCIA, ESPAÑA.

\section{Resumen.}

Antecedentes y objetivo: La fractura de cóndilo externo asociada a una luxación posteromedial de codo es una entidad muy rara, de la cual existen muy pocos casos publicados. Nuestro objetivo es presentar un caso complejo tratado en nuestro centro, el diagnóstico, el tratamiento elegido, y el seguimiento a las 9 semanas. Caso clínico: varón de 5 años con una luxación posteromedial de codo asociada a una fractura de cóndilo externo, diagnosticada mediante TC y tratada de forma quirúrgica mediante un abordaje anterior sobre el codo, reducción abierta y osteosíntesis con agujas de Kirschner. Resultados: los resultados clínicos y radiológicos a las 9 semanas son excelentes, presentando una consolidación radiográfica y un rango de movilidad articular excelente. Conclusiones: un diagnóstico y tratamiento quirúrgico precoz mediante reducción abierta y osteosíntesis del cóndilo externo es el tratamiento óptimo para estas lesiones, ya que una mala reducción deriva en malos resultados a largo plazo.

Palabras clave: luxación posteromedial; codo; cóndilo externo; infantil

\section{Summary.}

Background and aim: External humeral condyle fracture associated with a posteromedial elbow dislocation is a very rare entity, of which there are very few cases published. Our objective is to present a complex case treated in our Hospital, the diagnosis, the treatment we chose, and the follow-up at 9 weeks after the intervention. Clinical case: 5-year-old boy with a posteromedial elbow dislocation associated with an external humeral condyle fracture, which was diagnosed by CT and treated surgically using an anterior approach over the elbow, open reduction, and osteosynthesis with K-wires. Results: clinical and radiological results 9 weeks after the intervention were excellent, presenting a radiographic consolidation of the fracture and an excellent range of joint mobility. Conclusion: early diagnosis and surgical treatment through open reduction and osteosynthesis of the external condyle is the gold standard on treatment for these injuries, since a bad reduction leads to poor long-term results.

\section{Correspondencia:}

Álvaro Darás Ballester

E-mail: daras.alvaro@gmail.com

TIf: 685848548

Institución: Hospital Universitario y Politécnico La Fe, Valencia.

Dirección: Avenida de Fernando Abril Martorell, 106, 46026

Valencia, España 
DARÁS-BALLESTER A y COLS. Lesión compleja del codo pediátrico: fractura de cóndilo externo asociada a luxación posteromedial de codo.

\section{Introducción}

El codo es una de las articulaciones que más lesiones sufre durante la infancia. Las luxaciones de codo y las fracturas de cóndilo lateral son lesiones relativamente frecuentes en el codo pediátrico ${ }^{1-10}$. No obstante, la luxación posteromedial de codo asociada a una fractura de cóndilo externo del húmero es una lesión rara en la edad pediátrica, y de difícil diagnóstico inicial, debido a los múltiples núcleos de osificación que presenta el codo en la infancia $^{3-8}$. De hecho, no existe literatura en español sobre estas lesiones, y los casos publicados en lengua inglesa son escasos ${ }^{3,5,10}$. Generalmente, esta fractura se produce debido a una caída sobre la mano con semiflexión del codo, en posición de varo forzado ${ }^{3,4,7,8,9}$. El patrón típico de esta lesión incluye una luxación posteromedial del codo mientras el cóndilo externo queda alineado con la cabeza del radio $^{3,4}$. En este trabajo, presentamos un caso de luxación posteromedial de codo asociada a una fractura de cóndilo externo tipo II de Milch, su tratamiento y los resultados tanto clínicos como radiológicos a las 9 semanas de seguimiento, tras realizar una revisión de la literatura al respecto.

\section{Caso Clínico}

Varón de 5 años remitido desde otro hospital con el diagnóstico de fractura de cóndilo externo de húmero derecho, tras una caída con patinete en vía pública sobre la mano derecha. Llega a nuestro Hospital inmovilizado con una férula braquial con el codo en flexión de $90^{\circ}$.

No presenta antecedentes familiares ni personales de interés.

A la exploración física, se observa importante tumefacción y deformidad en cara externa del codo derecho (Fig. 1), impotencia funcional, y dolor difuso. Se constata la ausencia de lesiones cutáneas y la exploración neurológica y vascular es normal.

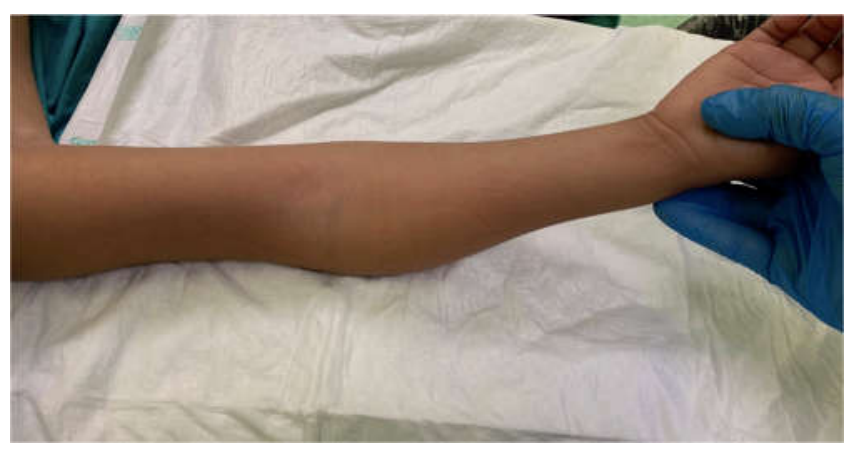

Figura 1: Exploración clínica inicial del paciente, donde se observa importante tumefacción a nivel de codo.

En las radiografías iniciales, realizadas en el centro desde el que se remite al paciente, se observa la fractura de cóndilo externo de codo derecho (Fig. 2).
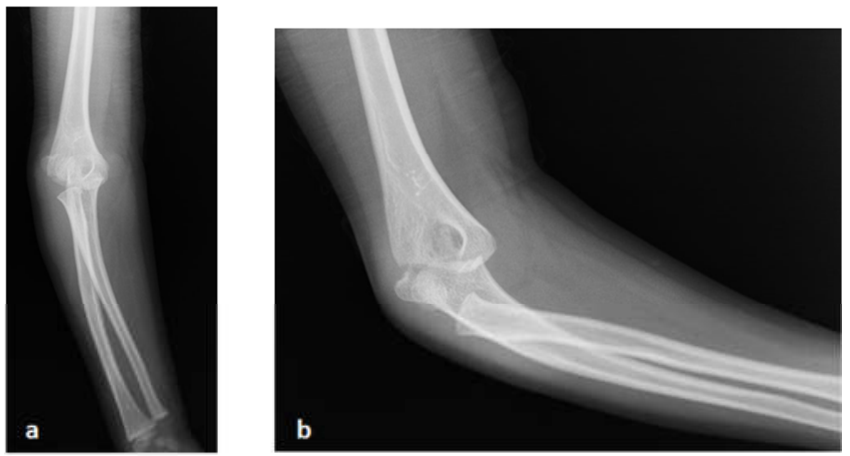

Figura 2: Radiografía anteroposterior (2.a) y lateral (2.b) de codo iniciales, que revelan una fractura de cóndilo externo humeral.

En nuestro hospital, se repiten las radiografías con la fractura inmovilizada mediante la férula braquial y se objetiva la presencia de una luxación posteromedial de codo asociada a la fractura de cóndilo humeral externo, que había pasado desapercibida en las radiografías iniciales (Fig. 3).
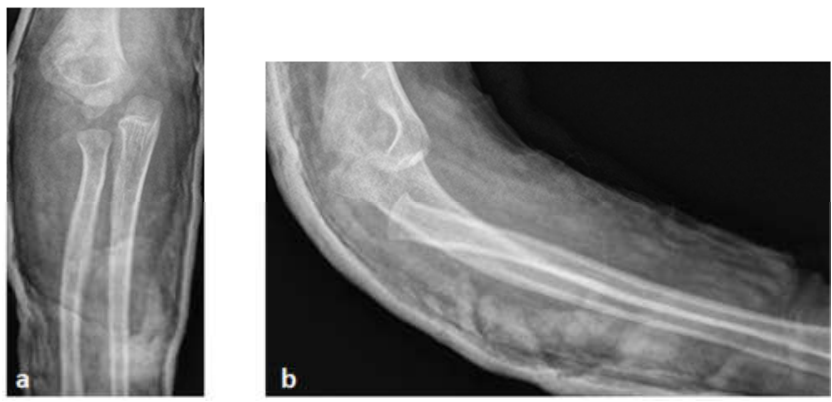

Figura 3: Radiografías anteroposterior (3.a) y lateral (3.b) de codo a la llegada a nuestro Hospital, donde se observa una luxación posteromedial de codo asociada a la fractura de cóndilo externo humeral.

A continuación, se realiza una Tomografía Computarizada (TC) para estudiar la lesión, que confirma la presencia de una luxación posteromedial de codo asociada a una fractura de cóndilo externo humeral, que mantiene la congruencia articular con la cabeza del radio (Fig. 4).

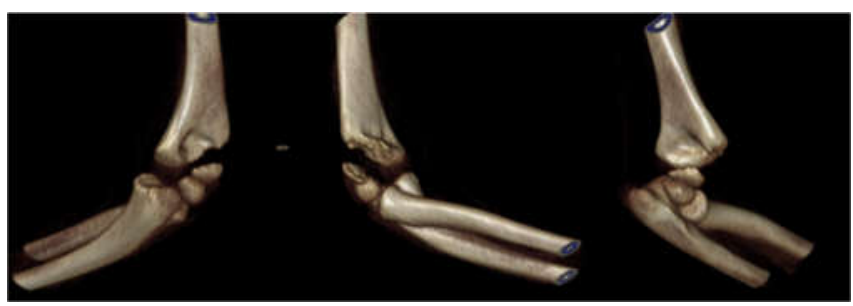

Figura 4: Reconstrucciones 3D de Tomografía Computarizada preoperatoria, en la cual se observa una luxación posteromedial de codo asociada a una fractura de cóndilo externo del húmero tipo II de la clasificación de Milch, que mantiene la congruencia articular con la cabeza del radio. 
DARÁS-BALLESTER A y COLS. Lesión compleja del codo pediátrico: fractura de cóndilo externo asociada a luxación posteromedial de codo.

El paciente se intervino, de forma programada a las 12 horas de evolución de la fractura: Bajo anestesia general, se comprobó la inestabilidad de la articulación con el varovalgo. Tras una reducción cerrada de la luxación, se colocó isquemia en la raíz del miembro superior derecho y se procedió a la reducción abierta y fijación interna de la fractura de cóndilo externo.

Para ello, se realizó un abordaje anterior de codo, mediante una incisión transversa sobre la flexura del codo (Fig. 5).

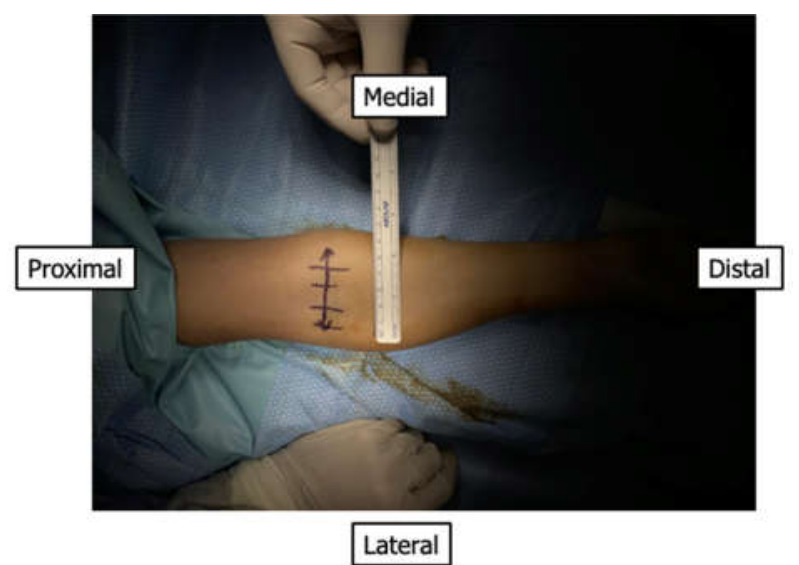

Figura 5: Planificación preoperatoria del abordaje anterior de codo.

Se identificaron el nervio mediano y la arteria braquial, se referenciaron y se protegieron, desplazándolos hacia medial. En la zona lateral, se identificó y referenció el nervio radial.

A continuación, se realizó una reducción abierta de la fractura (Figura 6) y se osteosintetizó con la colocación de 3 agujas de Kirschner de $1,8 \mathrm{~mm}$ desde lateral hacia medial (Fig. 7).
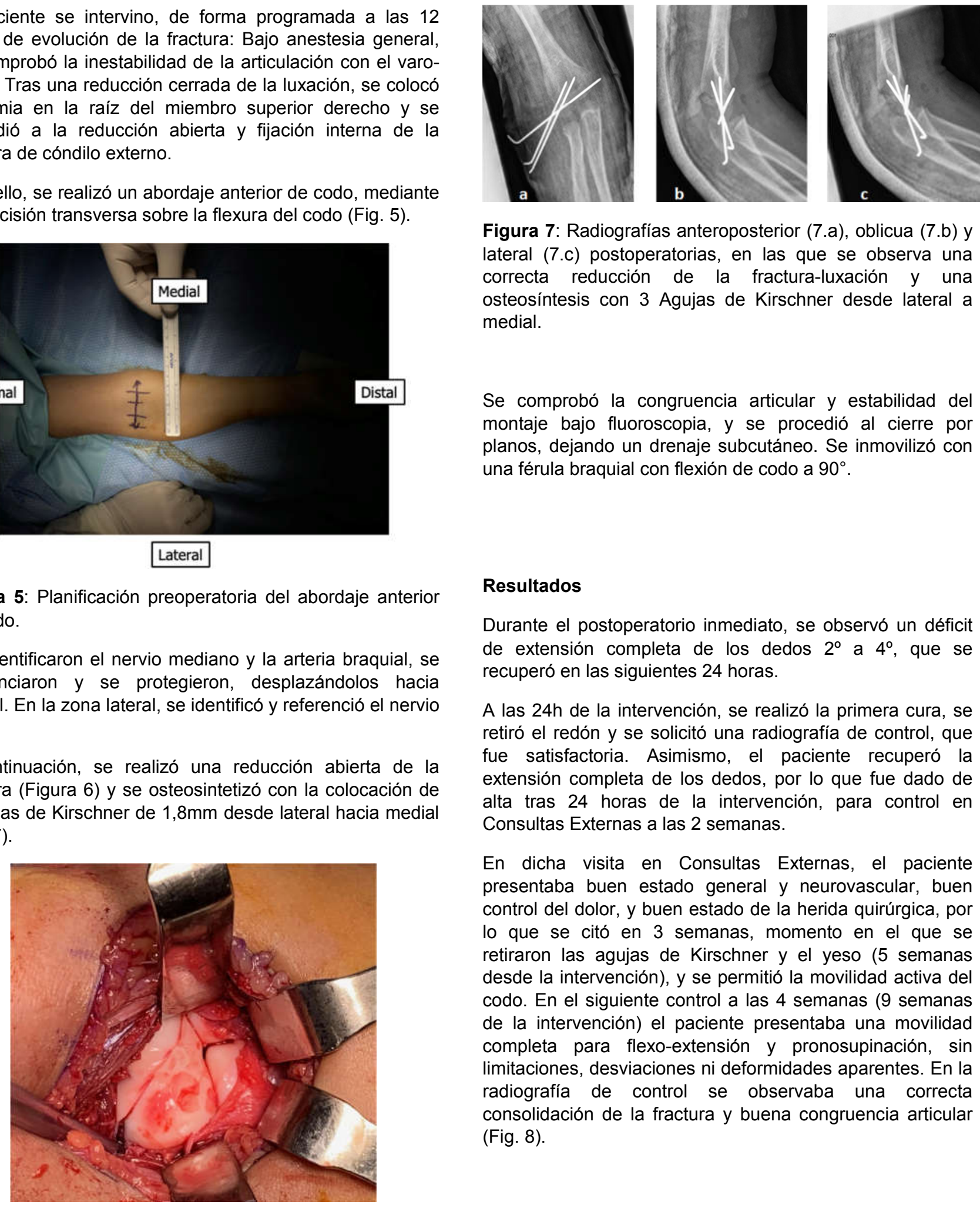

Figura 7: Radiografías anteroposterior (7.a), oblicua (7.b) y lateral (7.c) postoperatorias, en las que se observa una correcta reducción de la fractura-luxación y una osteosíntesis con 3 Agujas de Kirschner desde lateral a medial.

Se comprobó la congruencia articular y estabilidad del montaje bajo fluoroscopia, y se procedió al cierre por planos, dejando un drenaje subcutáneo. Se inmovilizó con una férula braquial con flexión de codo a $90^{\circ}$.

\section{Resultados}

Durante el postoperatorio inmediato, se observó un déficit de extensión completa de los dedos $2^{\circ}$ a $4^{\circ}$, que se recuperó en las siguientes 24 horas.

A las $24 \mathrm{~h}$ de la intervención, se realizó la primera cura, se retiró el redón y se solicitó una radiografía de control, que fue satisfactoria. Asimismo, el paciente recuperó la extensión completa de los dedos, por lo que fue dado de alta tras 24 horas de la intervención, para control en Consultas Externas a las 2 semanas.

En dicha visita en Consultas Externas, el paciente presentaba buen estado general y neurovascular, buen control del dolor, y buen estado de la herida quirúrgica, por lo que se citó en 3 semanas, momento en el que se retiraron las agujas de Kirschner y el yeso (5 semanas desde la intervención), y se permitió la movilidad activa del codo. En el siguiente control a las 4 semanas (9 semanas de la intervención) el paciente presentaba una movilidad completa para flexo-extensión y pronosupinación, sin limitaciones, desviaciones ni deformidades aparentes. En la radiografía de control se observaba una correcta consolidación de la fractura y buena congruencia articular (Fig. 8).

Figura 6: Reducción abierta de la fractura de cóndilo externo humeral por vía anterior, donde se observa la articulación radio-capitelar y trócleo-olecraniana 

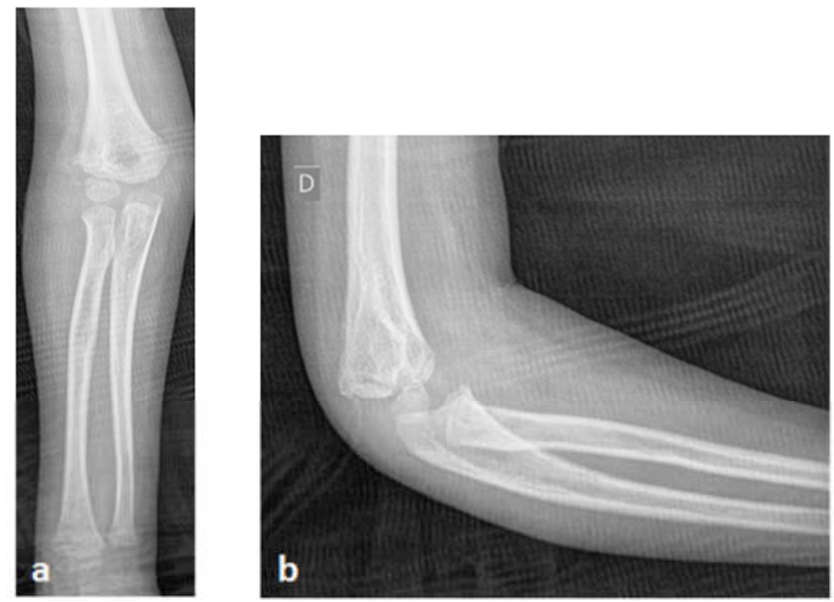

Figura 8: Radiografías anteroposterior (8.a) y lateral (8.b) de codo a las 9 semanas de la intervención, en las que se observa una correcta congruencia articular y consolidación de la fractura.

\section{Discusión}

Las fracturas de cóndilo externo de húmero son lesiones comunes en la edad pediátrica, pero la asociación de las mismas con una luxación posteromedial de codo ocurre en raras ocasiones ${ }^{3,4,5}$. El mecanismo que produce estas lesiones suele ser una caída sobre la mano al mismo tiempo que una fuerza aductora es ejercida sobre el codo en semiflexión y en varo ${ }^{3,4,7,8,9}$. Estas lesiones se presentan clínicamente como una importante deformidad observable a nivel del codo, limitación funcional y dolor. Se recomienda realizar radiografías con proyecciones anteroposterior y lateral del codo antes de realizar una reducción de la luxación para poder catalogar las lesiones, ya que es posible que después de la reducción, las líneas de fractura no se observen con claridad ${ }^{3,7,8}$. En muchos casos, es recomendable solicitar una TC para estudiar la lesión de cara a una planificación quirúrgica ${ }^{5}$.

Según la clasificación de Milch, las fracturas de cóndilo externo humeral de tipo II, en las que el foco de fractura afecta a la tróclea, se asocian con mayor frecuencia a luxaciones que las tipo $1^{3,7,8,9}$. Generalmente, lo que se observa en las pruebas de imagen es una luxación posteromedial del codo en la que el cóndilo externo humeral mantiene la congruencia articular con la cabeza del radio $^{3,7,8,9}$.

El diagnóstico temprano de estas lesiones, así como su tratamiento precoz es fundamental para el buen pronóstico de las mismas ${ }^{3,4,9,10}$. Según la literatura y nuestra propia experiencia, el método recomendado es una reducción cerrada de la luxación y una posterior reducción abierta y osteosíntesis de la fractura del cóndilo externo ${ }^{3,4,7,8,9}$. La reducción cerrada de la luxación es relativamente sencilla si no existen atrapamientos de partes blandas ${ }^{3,4,7,8}$. Muchos autores recomiendan la reducción abierta de la fractura de cóndilo externo mediante un abordaje lateral del mismo, y una osteosíntesis con 2 o 3 agujas de Kirschner de 1,6 o $1,8 \mathrm{~mm}^{3,5,7,8,9}$. No obstante, otros autores recomiendan el abordaje anterior de codo, ya que ofrece un mejor acceso a la articulación del codo, permite realizar una reducción anatómica de cara a obtener un mejor pronóstico, y evitar lesionar la vascularización del cóndilo lateral del húmero y por tanto, posibles complicaciones como la necrosis avascular o la pseudoartrosis ${ }^{10}$. En nuestro caso, se optó por este abordaje anterior. Tras la correcta reducción y osteosíntesis de la fractura, estas fracturas-luxaciones deben ser inmovilizadas con férula braquial durante al menos 4-6 semanas, tras las cuales se plantea la retirada de agujas y del yeso y el inicio de la movilización libre de la articulación ${ }^{4}$. En nuestro caso, los resultados clínicos y radiológicos fueron excelentes a las 9 semanas de seguimiento.

\section{Conclusiones}

La luxación posteromedial de codo asociada a una fractura de cóndilo externo del húmero es una lesión rara en la edad pediátrica. Cuando sospechamos esta entidad, la TC puede sernos de gran ayuda para caracterizar las lesiones. Respecto al tratamiento, según nuestra experiencia y nuestra revisión de la literatura al respecto, podemos recomendar la reducción cerrada de la luxación de codo, y la posterior reducción abierta y osteosíntesis de la fractura de cóndilo externo humeral, ya sea por abordaje lateral o anterior de codo. La precocidad en el diagnóstico y el tratamiento adecuado de la fractura-luxación, así como la correcta reducción de las mismas, son determinantes en cuanto al pronóstico, ya que una mala reducción puede resultar en pseudoartrosis o necrosis avascular del cóndilo externo humeral, así como en malos resultados funcionales. 
DARÁS-BALLESTER A y COLS. Lesión compleja del codo pediátrico: fractura de cóndilo externo asociada a luxación posteromedial de codo.

\section{Bibliografía}

1. Beaty JH, Kasser JR. El codo: fracturas fisarias, lesiones apofisarias del húmero distal, osteonecrosis avascular de la tróclea y fracturas condíleas en T. En: Beaty JH, Kasser JR. Rockwood and Wilkins' Fracturas en el niño. Madrid, España: Marbán Libros, S.L.; 2007, p. 625-704.

2. Thompson GH, et al. Luxaciones de codo. En: Beaty JH, Kasser JR. Rockwood and Wilkins' Fracturas en el niño. Madrid, España: Marbán Libros, S.L.; 200, p. 705-40.

3. Cheng PG, Chang WN, Wang MN. Posteromedial dislocation of the elbow with lateral condyle fracture in children. J Chin Med Assoc 2009; 72:103-7. https://doi.org/10.1016/S1726-4901(09)70033-4

4. Silva M, Cooper SD, Cha A. Elbow dislocation with an associated lateral condyle fracture of the humerus: a rare occurrence in the pediatric population. J Pediatr Orthop 2015; 35(4):329-33. https://doi.org/ 10.1097/BPO.0000000000000270

5. Gupta P, Sood M, Gupta S, Gupta R. Malunited condyle fracture of humerus with elbow dislocation: a diagnostic dilemma. European Journal of Orthopaedic Surgery and Traumatology 2018; 28:1441-5 https://doi.org/10.1007/s00590-018$2219-6$

6. Tachdjian MO. Fractures and dislocations: Pediatric Orthopaedics. 2nd ed. Philadelphia, PA: WB Saunders, 1990:3123-31

7. Wani I, Gupta N, Salaria A. Complex Elbow Injury In a 16 Year Old Boy: Medial Elbow Dislocation Associated With Lateral Condyle Fracture. The Internet Journal of Orthopedic Surgery 2007; 10(1):1-5.

8. Sharma H, Ayer R, Taylor GR. Complex pediatric elbow injury: an uncommon case. BMC Musculoskelet Disord 2005; 6:13 https://doi.org/10.1186/1471-2474-6-13

9. Lan X, Dai M, Zhang B, Huang G. Comparative study of lateral condyle fracture with or without posteromedial elbow dislocation in children. International Orthopaedics (SICOT) 2018; 42:619-24. https://doi.org/10.1007/s00264-018-3795-0

10. Tomori $Y$, Nanno M, Takai S. Posteromedial elbow dislocation with lateral humeral condyle fracture in children: three case reports and literature review. Medicine 2018; 97(36): e12182. https://doi.org/10.1097/MD.0000000000012182 